\title{
D-BRANES IN KAZAMA-SUZUKI MODELS
}

\author{
SONIA STANCIU
}

\begin{abstract}
We investigate boundary states of D-branes wrapped around supersymmetric cycles in Kazama-Suzuki models. We show that the geometry of the D-branes corresponds to a generalisation of calibrated geometry. We comment on the link with the geometry of the coset space and discuss how T-duality maps between these boundary states.
\end{abstract}

\section{INTRODUCTION}

The advent of D-branes forces us to reconsider the problem of consistent string vacua in order to determine the manifolds which allow consistent string propagation, both perturbative and non-perturbative. In particular, D-branes probe a new aspect of the background geometry, namely the geometry of submanifolds. For instance, the requirement that the worldvolume theory of a D-brane wrapping on a given submanifold of the compactification manifold be supersymmetric determines the so-called supersymmetric cycles, which in the case of backgrounds without a $B$ field $[1,2,3]$ are examples of calibrated submanifolds in the sense of $[4,5]$.

D-branes have been extensively studied in the case when their worldvolume is flat. However the study of D-branes on curved spaces (see for instance $[1,6,2,3,7,8,9,10])$ has been largely restricted to the case of Calabi-Yau manifolds and some manifolds of exceptional holonomy. Calabi-Yau spaces seem to be perfectly suited for the study of D-branes, as they provide us with much of the necessary information for this kind of analysis; however it is important to learn how much of it relies on exactly the Calabi-Yau structure, and how much remains true in slightly different or wider contexts.

Group manifolds and coset models, in particular the $N=2$ KazamaSuzuki models, are among the few known examples of explicit solutions for exact string backgrounds, and as such they make prime candidates for the study of D-branes in curved manifolds. Here the algebraic structure of the model imposes non-trivial consistency requirements on the possible boundary conditions [7]. However the essential feature that distinguishes these backgrounds from the ones based on CalabiYau manifolds is the presence, in this case, of a nontrivial $B$ field. One of the main purposes of this paper is to investigate how the geometry of

Supported by a PPARC Postdoctoral Fellowship. 
the supersymmetric cycles depends of this fact. Moreover in this case the geometric data is known exactly and we do not have to restrict ourselves to working in the large volume limit as is the case for CalabiYau manifolds.

In this paper we initiate a study of D-branes on coset manifolds by analysing, in the context of type II string theory, D-branes on KazamaSuzuki models. We therefore start in Section 2 by reviewing the basic aspects of the Kazama-Suzuki models, in order to set the notation and exhibit the superconformal structure of these models. In Section 3 we write down the consistency requirements that boundary states of $N=2$ superconformal theories (SCFT) have to satisfy. They determine two types of boundary conditions, much in the same way as happens in the case of Calabi-Yau threefolds [2]. In Section 4 we discuss a class of solutions for the case of the Kazama-Suzuki models. In Section 5 we examine the geometric interpretation of these boundary states in terms of D-branes wrapped around cycles in the coset space. The corresponding submanifolds saturate a bound which can be thought of as a geometric analogue of the Bogomol'nyi bound. The appropriate framework for describing these submanifolds is given by a generalisation [4] of the concept of calibration, which we discuss in Section 6. In general, these cycles are not volume-minimising, and we argue why this does not conflict with the requirement that they be BPS. As an application of these results, in Section 7 we discuss the way abelian $\mathrm{T}$-duality transformations map these D-brane configurations.

\section{KaZAMA-SuzuKi MODElS}

Let us start by defining our background. For this we consider a compact simple Lie group $G$, and $H$ a subgroup with $\mathfrak{h} \subset \mathfrak{g}$ the corresponding Lie algebras. By the Kazama-Suzuki model [11, 12, 13] we mean the $N=2$ superconformal field theory constructed from the $N=1$ coset theory $G / H$. In order to define this model we need an $N=1$ affine algebra $\widehat{\mathfrak{g}}_{N=1}$, with generators $\left(I_{a}, \Psi_{a}\right)$ and

$$
\begin{aligned}
I_{a}(z) I_{b}(w) & =\frac{k \Omega_{a b}}{(z-w)^{2}}+\frac{f_{a b}^{c} I_{c}(w)}{z-w}+\mathrm{reg}, \\
I_{a}(z) \Psi_{b}(w) & =\frac{f_{a b}{ }^{c} \Psi_{c}(w)}{z-w}+\mathrm{reg}, \\
\Psi_{a}(z) \Psi_{b}(w) & =\frac{k \Omega_{a b}}{z-w}+\mathrm{reg} .
\end{aligned}
$$

$\Omega$ denotes an invariant metric of $\mathfrak{g}$ and the parameter $k$ is related to the level $x$ of the affine algebra by $k=x+g^{*}$, where $g^{*}$ is the dual Coxeter number. Further we need an $N=1$ affine subalgebra $\widehat{\mathfrak{h}}$ of $\widehat{\mathfrak{g}}$, generated by the currents $\left(I_{i}, \Psi_{i}\right)$, and whose OPEs are similar to (1) -(3), with metric $\Omega_{i j}$, the restriction $\left.\Omega\right|_{\mathfrak{h}}$ of $\Omega$ to $\mathfrak{h} \subset \mathfrak{g}$. 
For the coset theory to have $N=2$ superconformal symmetry, $\mathfrak{g} / \mathfrak{h}$ must possess an $\mathfrak{h}$-invariant metric $\Omega_{\alpha \beta}$, and a compatible, integrable, $\mathfrak{h}$-invariant complex structure $A_{\alpha}{ }^{\beta}$ (see [14] for details). Then the generators of the $N=2 \mathrm{SCA}$ will be given by

$$
\begin{aligned}
\mathrm{T}(z)= & \frac{1}{2 k} \Omega^{\alpha \beta}\left(J_{\alpha} J_{\beta}\right)+\frac{1}{2 k}\left(\Omega^{\alpha \beta}+\frac{1}{k} f^{\alpha}{ }_{i \gamma} f^{i \gamma \beta}\right)\left(\partial \Psi_{\alpha} \Psi_{\beta}\right) \\
& +\frac{1}{2 k^{2}} f^{i \alpha \beta}\left(J_{i} \Psi_{\alpha} \Psi_{\beta}\right)-\frac{1}{8 k^{3}} f_{i}{ }^{\gamma \delta} f^{i \alpha \beta}\left(\Psi_{\alpha} \Psi_{\beta} \Psi_{\gamma} \Psi_{\delta}\right), \\
\mathrm{G}_{0}(z)= & \frac{1}{k} \Omega^{\alpha \beta}\left(J_{\alpha} \Psi_{\beta}\right)-\frac{1}{6 k^{2}} f^{\alpha \beta \gamma}\left(\Psi_{\alpha} \Psi_{\beta} \Psi_{\gamma}\right), \\
\mathrm{G}_{1}(z)= & \frac{1}{k} A^{\alpha \beta}\left(J_{\alpha} \Psi_{\beta}\right)+\frac{1}{6 k^{2}} B^{\alpha \beta \gamma}\left(\Psi_{\alpha} \Psi_{\beta} \Psi_{\gamma}\right), \\
\mathrm{J}(z)= & \frac{1}{2 i k} A^{\alpha \beta}\left(\Psi_{\alpha} \Psi_{\beta}\right)-\frac{1}{2 i k} A^{\gamma \delta} f_{\gamma \delta} I_{c},
\end{aligned}
$$

where $J_{a} \equiv I_{a}-\frac{1}{2 k} \Omega^{b d} f_{a b}^{c}\left(\Psi_{c} \Psi_{d}\right)$ and $B^{\mu \nu \rho}=A^{\mu \alpha} A^{\nu \beta} A^{\rho \gamma} f_{\alpha \beta \gamma}$. Notice that although so far we have only considered the holomorphic sector, we have a similar structure for the antiholomorphic sector as well. In other words, we have a $(2,2)$ SCFT.

Finally, we recall that one can describe the complex structure on $\mathfrak{g} / \mathfrak{h}$ in an alternative way, by introducing the projection operators $\left(P^{ \pm}\right)_{\beta}^{\alpha}=$ $\frac{1}{2}\left(\delta_{\beta}^{\alpha} \pm \frac{1}{i} A_{\beta}^{\alpha}\right)$, which allow us to split the complexified tangent space $\mathfrak{t} \cong$ $(\mathfrak{g} / \mathfrak{h})^{\mathbb{C}}$ into subspaces $\mathfrak{t}_{+}$and $\mathfrak{t}_{-}$defined as the image of the projectors $P^{+}$and $P^{-}$respectively. Introducing bases $\left\{X_{\alpha}^{ \pm}=\left(P^{ \pm}\right)_{\alpha}^{\beta} X_{\beta}\right\}$ for $\mathfrak{t}_{ \pm}$respectively, one can then show that $\mathfrak{t}_{ \pm}$are lagrangian, and that $\mathfrak{t}$ admits a decomposition $\mathfrak{t}=\mathfrak{t}_{+} \oplus \mathfrak{t}_{-}$into subspaces which close under the Lie brackets: $\left[\mathfrak{t}_{ \pm}, \mathfrak{t}_{ \pm}\right] \subset \mathfrak{t}_{ \pm}$(which re-states the fact that the complex structure on $G / H$ is integrable).

\section{BOUNDARY CONDITIONS FOR $N=2$ SCFTs}

D-branes can be been studied in a variety of ways: by using the techniques of perturbative string theory, they can be described either in terms of boundary conditions of open strings or as boundary states in the closed string sector. The concept of a boundary state, which describes how closed strings are emitted or absorbed on the D-brane worldvolume, allows us to perform perturbative string computations in the presence of a D-brane and is thus instrumental in considering D-branes in type II string theories.

The guiding principle behind constructing a boundary state is conformal invariance. In open string theories one has to impose constraints on the boundary conditions such that the (super)conformal symmetry is not broken. Then the boundary can be thought of as a closed string state where the left-and right-moving (super)conformal structures are related in a consistent way. Consistency means in this context that the holomorphic SCFT is set equal to the antiholomorphic SCFT, up to an automorphism of the $N=2 \mathrm{SCA}$. In other words

$$
\mathrm{A}(z)=\tau(\overline{\mathrm{A}}(\bar{z}))
$$


where $\mathrm{A}$ is a generic $N=2$ generator and $\tau$ is an arbitrary element of the automorphism group of the $N=2 \mathrm{SCA}$, that is $O(2)$. This ensures that the D-brane configuration preserves one set of the $N=2 \mathrm{SCA}$.

Further we require that our boundary state will possess local $N=1$ worldsheet supersymmetry. This means that the automorphism defining the boundary conditions has to fix the $N=1$ subalgebra generated by $\left(T, G_{0}\right)$, which leaves us with a $\mathbb{Z}_{2} \times \mathbb{Z}_{2}$ group of transformations.

Finally we want the D-brane configuration to describe a BPS state, and hence to preserve half of the spacetime supersymmetry. This forces us to extend the boundary conditions to the spectral flow operator. In the case of an $N=2 \mathrm{SCFT}$ spacetime supersymmetry is directly related to the $U(1)$ current [15]. Indeed if one considers the bosonisation of the $U(1)$ generator

$$
\mathrm{J}(z)=i \partial \phi
$$

then the spectral flow operator will be given by

$$
X(z)=e^{i \phi} .
$$

This leaves us with the following sets of boundary conditions:

(i) A-type boundary conditions

$$
\begin{gathered}
\mathrm{T}=\overline{\mathrm{T}}, \quad \mathrm{G}_{0}= \pm \overline{\mathrm{G}}_{0} \\
\mathrm{~J}=-\overline{\mathrm{J}}, \quad \mathrm{G}_{1}=\mp \overline{\mathrm{G}}_{1} \\
e^{ \pm i \phi}=e^{ \pm i \theta} e^{\mp i \bar{\phi}}
\end{gathered}
$$

(ii) B-type boundary conditions

$$
\begin{gathered}
\mathrm{T}=\overline{\mathrm{T}}, \quad \mathrm{G}_{0}= \pm \overline{\mathrm{G}}_{0}, \\
\mathrm{~J}=\overline{\mathrm{J}}, \quad \mathrm{G}_{1}= \pm \overline{\mathrm{G}}_{1}, \\
e^{ \pm i \phi}=e^{ \pm i \theta} e^{ \pm i \bar{\phi}} .
\end{gathered}
$$

These boundary conditions have been written down previously in [2], in the context of Calabi-Yau compactifications. However they are clearly valid for a generic $N=2$ theory, independent of any particular model. In order to find solutions for them we will have to specify a particular class of SCFTs - in our case the Kazama-Suzuki model.

\section{Boundary CONDitions for KazAMA-Suzuki MOdelS}

The first step in solving our problem is finding a consistent set of boundary conditions for the current algebra (1)-(3) in terms of which the Kazama-Suzuki model is defined, such that one of the two possible types of boundary states will be realised. (Alternatively one could argue that the boundary conditions for the $N=2$ SCA are not restrictive enough to determine the allowed configurations uniquely.) This 
approach to constructing boundary states, which goes back to [16], was used recently in [7] for the study of bosonic D-branes on group manifolds.

Thus we require that the boundary conditions satisfied by the bosonic and fermionic currents

$$
I_{a}(z)=R_{a}^{b} \bar{I}_{b}(\bar{z}), \quad \Psi_{a}(z)=S_{a}^{b} \bar{\Psi}_{b}(\bar{z}),
$$

preserve the $N=1$ affine algebra (1)-(3), which imposes the following conditions on the matrices $R$ and $S$ :

$$
\begin{aligned}
R_{a}{ }^{c} R_{b}{ }^{d} \Omega_{c d} & =\Omega_{a b}, & & R_{a}{ }^{d} R_{b}{ }^{e} f_{d e}{ }^{f}=f_{a b}{ }^{c} R_{c}{ }^{f}, \\
S_{a}{ }^{c} S_{b}{ }^{d} \Omega_{c d} & =\Omega_{a b}, & & R_{a}{ }^{d} S_{b}{ }^{e} f_{d e}{ }^{f}=f_{a b}{ }^{c} S_{c}{ }^{f} .
\end{aligned}
$$

This immediately implies that the $N=1$ Sugawara energy-momentum tensor $\mathrm{T}_{\mathfrak{g}}(z)=\frac{1}{2 k} \Omega^{a b}\left(J_{a} J_{b}\right)+\frac{1}{2 k} \Omega^{a b}\left(\partial \Psi_{a} \Psi_{b}\right)$ constructed from $\widehat{\mathfrak{g}}_{N=1}$ satisfies

$$
\mathrm{T}_{\mathfrak{g}}=\overline{\mathrm{T}}_{\mathfrak{g}},
$$

at the boundary. From the boundary condition on $\mathrm{G}_{0}$ one deduces that $\Omega^{\alpha \beta} R_{\alpha}{ }^{i} S_{\beta}{ }^{a}=\Omega^{\alpha \beta} R_{\alpha}{ }^{a} S_{\beta}{ }^{i}=0$ which in turn implies that

$$
R_{\alpha}{ }^{i}=S_{\alpha}{ }^{i}=0 \text {. }
$$

In other words $R$ and $S$ preserve $\mathfrak{h}$ and $\mathfrak{g} / \mathfrak{h}$. Moreover, from (16) and (17) and by using Schur's lemma we deduce that

$$
S= \pm R
$$

as one would expect from supersymmetry.

On the other hand (19) implies that the $N=1$ Sugawara energymomentum tensor $\mathrm{T}_{\mathfrak{h}}(z)=\frac{1}{2 k} \Omega^{i j}\left(J_{i} J_{j}\right)+\frac{1}{2 k} \Omega^{i j}\left(\partial \Psi_{i} \Psi_{j}\right)$ constructed from $\widehat{\mathfrak{h}}_{N=1}$ satisfies

$$
\mathrm{T}_{\mathfrak{h}}=\overline{\mathrm{T}}_{\mathfrak{h}}
$$

which together with (18) ensures that that boundary condition corresponding to the Kazama-Suzuki energy-momentum tensor $\mathrm{T}$ is satisfied. Similarly one can also show for the other $N=1$ Sugawara generators

$$
\left(\mathrm{G}_{0}\right)_{\mathfrak{g}}= \pm\left(\overline{\mathrm{G}}_{0}\right)_{\mathfrak{g}}, \quad\left(\mathrm{G}_{0}\right)_{\mathfrak{h}}= \pm\left(\overline{\mathrm{G}}_{0}\right)_{\mathfrak{h}},
$$

which implies that the boundary conditions for the $\mathfrak{g}$ and $\mathfrak{h} N=1$ SCAs are independently satisfied.

We now turn to the boundary conditions for $G_{0}$, which are the same for both types $\mathrm{A}$ and $\mathrm{B}$; they impose the following conditions:

$$
\begin{aligned}
R_{\alpha}{ }^{\gamma} R_{\beta}{ }^{\delta} \Omega_{\gamma \delta} & =\Omega_{\alpha \beta}, \\
R_{\alpha}{ }^{\mu} R_{\beta}{ }^{\nu} R_{\gamma}{ }^{\rho} f^{\alpha \beta \gamma} & =f^{\mu \nu \rho} .
\end{aligned}
$$

Hence, according to the first relation, $R$ preserves the metric on $\mathfrak{g} / \mathfrak{h}$. 
The boundary conditions on $\mathrm{G}_{1}$, on the other hand are slightly different for the two types of boundary conditions, and relate $R$ to the complex structure on $G / H$ :

$$
\begin{aligned}
A^{\alpha \beta} R_{\alpha}{ }^{\gamma} R_{\beta}{ }^{\delta} & =\mp A^{\gamma \delta}, \\
B^{\alpha \beta \gamma} R_{\alpha}{ }^{\mu} R_{\beta}{ }^{\nu} R_{\gamma}{ }^{\rho} & =\mp B^{\mu \nu \rho},
\end{aligned}
$$

where the two signs refer to the A and B types of boundary conditions, respectively. It is easy to see that the second condition (24) follows from (22) and (23), hence it does not yield any new information. On the other hand, the first condition (23) can be rephrased by saying that the complex structure $A$ (anti)commutes with the matrix $R$. If we now consider the split of $\mathfrak{t}$ into the subspaces $\mathfrak{t}_{+}$and $\mathfrak{t}_{-}$, then (23) amounts to two sets of boundary conditions (one for each case) for the generators of these subspaces: $X_{\alpha}^{ \pm}=R_{\alpha}{ }^{\beta} \bar{X}_{\beta}^{\mp}$ for the A-type, and $X_{\alpha}^{ \pm}=$ $R_{\alpha}{ }^{\beta} \bar{X}_{\beta}^{ \pm}$for the B-type boundary conditions where, in order to avoid confusion, we denoted by $\left\{\bar{X}_{\alpha}\right\}$ the generators of $\mathfrak{g} / \mathfrak{h}$ corresponding to the antiholomorphic sector. Symbolically we can write this conditions as $\mathfrak{t}_{ \pm}=R\left(\overline{\mathfrak{t}}_{\mp}\right)$ and $\mathfrak{t}_{ \pm}=R\left(\overline{\mathfrak{t}}_{ \pm}\right)$, respectively.

One can check that the boundary conditions on the $U(1)$ current $\mathrm{J}$ do not provide any new conditions relating $R$ to the geometric data of the target space. Therefore from (8) one can deduce that $\phi=\mp \bar{\phi}+\theta$, where $\theta$ is a constant. However in order to properly analyse the boundary conditions (12) and (15), and in particular to fix $\theta$, one would need an explicit expression of the spectral flow (9) in terms of the affine currents (which are $N=1$ primaries) and the geometric data on the coset space.

\section{Supersymmetric CyCLES}

We will study here the geometry of the supersymmetric cycles in Kazama-Suzuki models. By a configuration in which a D-brane wraps around one such cycle, we mean one in which we identify the worldvolume of the D-brane with the cycle or, more precisely, we identify the tangent and normal directions to the cycle with the tangent and normal directions to the worldvolume of the D-brane, respectively. ${ }^{1}$ We will restrict ourselves to the case without mixed boundary conditions. In this case the matrix $R$ is diagonalisable, with the eigenvectors corresponding to the $( \pm 1)$-eigenvalues being identified with the Neumann and Dirichlet boundary conditions on the bosonic currents, respectively.

It is easy to see that the Neumann directions are indeed tangent to submanifolds of $G / H$; that is, to the D-branes. We see this first at

\footnotetext{
${ }^{1}$ Strictly speaking we mean the component of the worldvolume of the D-brane in the internal theory defined by the Kazama-Suzuki model. From the point of view of an observer in the spacetime, such a D-brane configuration could present itself point-like, if the D-brane has no tangent directions in the spacetime, or extended, if it does. For euclidean D-branes, it could even present itself as an instanton. Our discussion clearly does not depend on these details.
} 
a point in $G / H$ (the identity coset), where we can identify the tangent space with $\mathfrak{g} / \mathfrak{h}$. The Neumann directions correspond to the $(+1)$ eigenvalues of the matrix $R$; let us call them $(\mathfrak{g} / \mathfrak{h})_{+}$. We would like to interpret $(\mathfrak{g} / \mathfrak{h})_{+}$as the tangent space of a submanifold of $G / H$ at that point. This would follow from the Frobenius integrability theorem if we could show that vector fields tangent to the Neumann directions close under the Lie bracket. Let us prove this. Under the action of $R$, $\mathfrak{g}$ also decomposes as $\mathfrak{g}=\mathfrak{g}_{+} \oplus \mathfrak{g}_{-}$, where $\mathfrak{g}_{+}$is a subalgebra. Because $R$ is block diagonal, it follows that $(\mathfrak{g} / \mathfrak{h})_{+}$is the projection of $\mathfrak{g}_{+}$onto $\mathfrak{g} / \mathfrak{h}$. The projection $\mathfrak{g} \rightarrow \mathfrak{g} / \mathfrak{h}$ extends to a Lie algebra homomorphism from $\mathfrak{g}$ to the vector fields on $G / H$. The image of this map are the fundamental (or Killing) vectors generating infinitesimally the $G$ action. Because $\mathfrak{g}_{+}$is a subalgebra, its image closes under Lie brackets. As a matter of fact, Frobenius's theorem tells us more: it guarantees that every point in $G / H$ is contained in a unique submanifold whose tangent vectors are the Killing vectors in $\mathfrak{g}_{+}$; that is, $G / H$ is foliated by its D-branes.

This raises an interesting question; namely, whether this foliation of the coset space by its D-branes bears any similarity to the foliation of a Calabi-Yau space by its special lagrangian tori $[17,18]$. One could go even further and speculate about a possible generalisation of the mirror conjecture of [17] in the context of coset spaces. The answer to these questions is beyond our reach at the present moment, largely due to our insufficient understanding of the geometry of coset spaces, in particular of their calibrated submanifolds (as we will see in a moment). However we should point out that the origin of the two foliations is slightly different: in the case of $G / H$ it is a consequence of the homogeneity of the coset manifold, by contrast to Calabi-Yau spaces which are far from being homogeneous.

Let us now consider an $n$-cycle $\gamma$ in our target space. We want to study the configurations corresponding to D-branes wrapping around this cycle. Locally we can choose coordinates (that is, a basis for $\mathfrak{g} / \mathfrak{h})$ such that $\alpha=1, \ldots, n$ corresponds to the directions tangent to $\gamma$, whereas $\mu=n+1, \ldots, \operatorname{dim} G / H$ corresponds to the directions normal to the cycle. We then have two possible situations depending on the type of boundary state to which the D-brane gives rise, when wrapped around $\gamma$.

A-type cycles. The D-brane wrapped around $\gamma$ gives rise to a boundary state with type A boundary conditions. Then the condition (21), coming from the boundary condition satisfied by $\mathrm{G}_{0}$, implies that the metric $\Omega$ on $\mathfrak{g} / \mathfrak{h}$ has a block diagonal form with respect to $\gamma$, that is $\Omega_{\alpha \mu}=0$, which we can write symbolically as follows:

$$
\Omega=\Omega_{\gamma}+\Omega_{\gamma^{\perp}}
$$


In other words the metric on our coset space $G / H$ naturally induces a metric $\Omega_{\gamma}$ on the cycle.

The boundary condition on $\mathrm{G}_{1}$, which for the A-type cycles is given by (23) with the minus sign, implies that the 2 -form $A$ defined by

$$
A\left(X_{\alpha}, X_{\beta}\right)=A_{\alpha \beta}
$$

vanishes when restricted to either $\gamma$ or $\gamma^{\perp}$. Since $A$ is nondegenerate, it follows that the dimension of the cycle has to be equal to half the dimension of $G / H$, that is

$$
n=\frac{1}{2} \operatorname{dim} G / H .
$$

The vanishing of $A$ means that the complex structure maps vectors tangent to $\gamma$ to vectors tangent to $\gamma^{\perp}$; hence the tangent space to $\gamma$ contains no complex lines. In other words, it is a totally real subspace of $\mathfrak{g} / \mathfrak{h}$. By analogy with symplectic geometry we will say that $\gamma$ is a lagrangian submanifold; but it is important to emphasise that $A$ need not be closed. Indeed, using the integrability condition for the complex structure one can show that

$$
d A\left(X_{\alpha}, X_{\beta}, X_{\gamma}\right)=-B_{\alpha \beta \gamma} .
$$

Hence $d A=0$ if and only if $B_{\alpha \beta \gamma}=0$ which, given that $A$ is nondegenerate, is equivalent to $f_{\alpha \beta \gamma}=0$. We therefore conclude that the 2-form $A$ is a Kähler form on $G / H$ if and only if $G / H$ is a hermitian symmetric space (HSS). This does not mean that $G / H$ non-symmetric might not possess a $G$-invariant Kähler structure. Indeed, a theorem of Borel [19] guarantees that this is the case for $G$ and $H$ compact, provided $H$ is the centraliser of a torus.

B-type cycles. We now consider a D-brane that wraps on a cycle $\gamma$ giving rise to a boundary state of type $B$. Since $G_{0}$ satisfies the same boundary conditions as before we are lead to the same conclusion (25) about the metric on the coset space. On the other hand, the boundary conditions (23) (now with the plus sign) will imply that $A$ has a block diagonal structure with respect to $\gamma$, that is $A_{\alpha \mu}=0$, which we can write symbolically as

$$
A=A_{\gamma}+A_{\gamma^{\perp}} .
$$

Since $A$ is nondegenerate it follows that both $A_{\gamma}$ and $A_{\gamma^{\perp}}$ are nondegenerate as well. Furthermore it follows that the complex structure on $G / H$ induces an almost complex structure on the cycle. One can in fact show that $A_{\gamma}$ satisfies the integrability condition. Indeed, if we recall [14] that the integrability condition for the complex structure on $G / H$ (which is equivalent to the vanishing of its Nijenhuis tensor) reads

$$
f^{\mu \nu \rho}-A^{\mu \alpha} A^{\nu \beta} f_{\alpha \beta}{ }^{\rho}-A^{\nu \alpha} A^{\rho \beta} f_{\alpha \beta}{ }^{\mu}-A^{\rho \alpha} A^{\mu \beta} f_{\alpha \beta}{ }^{\nu}=0,
$$


we can easily deduce that also $A_{\gamma}$ satisfies such a condition and hence that it defines a complex structure on $\gamma$. In other words, $\gamma$ is a complex submanifold of $G / H$. In particular, its dimension must be even ${ }^{2}$

$$
\begin{aligned}
& n=0,2,4, \ldots, \operatorname{dim} G / H . \\
& \text { 6. D-BRANE GEOMETRY }
\end{aligned}
$$

In the previous section we have determined the geometric characteristics of D-branes on Kazama-Suzuki models with target space a homogeneous hermitian manifold $G / H$. The two types of boundary conditions give rise to two types of submanifolds:

(A) lagrangian submanifolds, and

(B) complex submanifolds.

At first sight this looks very similar to the case of Calabi-Yau manifolds, in which one gets special lagrangian and Kähler submanifolds, respectively $[1,2]$; these submanifolds are distinguished in that they are examples of calibrated submanifolds. In the case of coset models, the above submanifolds are not calibrated - in fact, they need not be minimal - but they will be seen to belong to a class of geometries which are not unrelated to calibrated geometries. In order to explain this, it is necessary to digress slightly into the subject of calibrated geometries $[4]$.

There is more than one way to formulate the geometric structure of a manifold. Perhaps the most common approach is to specify such a structure through a distinguished family of tensor fields on the manifold, e.g., the Kähler form; or by restricting the types of coordinate transformations which are allowed between different charts, e.g., Gstructures. A less common approach, but one which seems particularly relevant for the study of D-branes, is to specify a distinguished family of submanifolds. One way of singling out a family of submanifolds is via the method of calibrations.

A calibration in a riemannian manifold $M$ is a closed $p$-form $\omega$ which, acting on any unit simple $p$-vector $\xi$ in $\bigwedge^{p} T_{x} M$, satisfies $\omega(\xi) \leq 1$. In other words, the restriction of $\omega$ to any $p$-plane is less than or equal to the volume element. A $p$-plane is said to be calibrated by $\omega$ if $\omega$ coincides with the volume element. A $p$-dimensional submanifold $N$ is said to be calibrated by $\omega$ if its tangent space $T_{x} N$ is calibrated by $\omega_{x}$ for all $x$ in $N$. Calibrated submanifolds minimise volume in their homology class. Indeed, if $N^{\prime}$ is any other $p$-dimensional submanifold homologous to $N$, then

$$
\operatorname{vol} N=\int_{N} \omega=\int_{N^{\prime}} \omega \leq \operatorname{vol} N^{\prime}
$$

2 The nomenclature can be slightly misleading here since the B-type boundary conditions seem to describe even-dimensional D-branes which, on the other hand, appear in type IIA theories. 
where the second equality uses Stokes' theorem and the fact that $\omega$ is closed.

This concept has an interesting generalisation, which is called a $\phi$ geometry [4]. This is very similar to a calibrated geometry, the only difference being that the form $\phi$ need not be closed. This difference has as a consequence the fact that the associated "calibrated" submanifolds are not globally volume-minimising.

On a compact manifold any closed form can be normalised so that it is a calibration, but the computation of the normalisation is a very difficult problem and so is the determination of the calibrated submanifolds. Luckily some types of manifolds come with ready-made calibrations. For example any complex submanifold of a Kähler manifold is calibrated by the relevant power of the Kähler form; also special lagrangian submanifolds of Calabi-Yau manifolds are calibrated by the real part of the holomorphic volume form, whereas associative, coassociative and Cayley submanifolds of manifolds with exceptional holonomy are also calibrated by parallel forms naturally appearing in those geometries.

Kähler and special lagrangian submanifolds of a Calabi-Yau manifold made their appearance in $[1,2]$ in the context of D-branes. More precisely, it was shown that the worldvolume theory of a euclidean D-brane is supersymmetric precisely when the submanifold on which the D-brane wraps is either Kähler or special lagrangian. The volume minimisation property for these manifolds is then the geometric restatement of the BPS condition. This result is consistent with the dynamics of euclidean D-branes, in that when the $B$-field and the gauge field on the worldvolume of the D-brane are turned off, the Dirac-Born-Infeld action is simply the volume of the D-brane.

In the case of Kazama-Suzuki models $G / H$, we have found that the B-type cycles are complex submanifolds relative to the $G$-invariant complex structure. Wirtinger's inequality still applies and we can conclude that the tangent planes to these submanifolds are calibrated with respect to the normalised power of the nondegenerate 2 -form $A$. However, because $A$ is not closed in general, these submanifolds fail to be minimal, and what we have is a complex geometry defined by $A$.

It is important to remark that this result does not conflict with the requirement that these configurations be BPS. We have seen that the D-branes in Kazama-Suzuki models are not necessarily minimal submanifolds, and this conclusion seems to run against our geometric intuition, which leads us to expect that supersymmetric cycles be calibrated or at least minimal. However this expectation is born out of the assumption that the mass of a BPS state obtained by a D-brane wrapped on a supersymmetric cycle is given by its volume. In the cases where this has been shown to hold, the string couples only to the background metric; but it is hard to argue convincingly that the mass 
of the D-brane should not change in the presence of other background fields: the $B$-field or the dilaton, for instance (although see $[20,21]$ ). In the case of the Kazama-Suzuki model, the string couples to the $B$ field (whose torsion is given by the structure constants of $\mathfrak{g}$ ) and hence, strictly speaking, we cannot conclude that a BPS state, while certainly having minimal mass, also has minimal volume.

In the special case of $G / H$ a hermitian symmetric space the $B$ field is absent (being essentially the torsion of the $H$-connection, which vanishes in the symmetric case). But in this case, $A$ is a closed form, and the submanifolds calibrated by powers of $A$ are indeed volume minimising.

For the A-type cycles the situation is much less clear, due to the fact that, lacking an explicit form for the spectral flow generator, one is missing one extra condition. One therefore expects that A-type supersymmetric cycles will not just be lagrangian, but will be somehow further constrained. With the Calabi-Yau example in mind, one would hope that a condition akin to the special lagrangian condition should hold.

The problem of determining whether a homogeneous hermitian space $G / H$ possesses both A-type and B-type cycles is not an easy one. B-type cycles certainly always exist: $G / H$ itself is a complex submanifold albeit not a proper one. The situation for A-type cycles is not as clear. First of all it is easy to see that there are hermitian symmetric spaces which do not possess nontrivial lagrangian submanifolds; e.g., the 2 -sphere $S^{2} \cong S U(2) / U(1)$. The two-sphere is a Kähler manifold which possesses zero- and two-dimensional complex submanifolds and one-dimensional lagrangian submanifolds (isomorphic to $S^{1}$ ). However, whereas the complex submanifolds are indeed nontrivial calibrated cycles, $S^{1}$ is not calibrated and in fact it is a trivial cycle. This is symptomatic of a more general situation: For compact homogeneous Kähler spaces the odd cohomology groups are trivial [19], and this implies that for coset spaces of dimension $4 k+2$ there can be no nontrivial middle-dimensional cycles. On the other hand, when $\operatorname{dim} G / H=4 k$, both types of cycles are even-dimensional; however finding nontrivial lagrangian cycles for a given coset space is a difficult task. (In fact even for Calabi-Yau spaces, which have been extensively studied, very few explicit examples of special lagrangian submanifolds are known [18].) Nevertheless they do exist. For example, the coset $S U(2+2 k) / S(U(2) \times U(2 k))$, which is an $8 k$-dimensional hermitian symmetric space of type AIII in Helgason's nomenclature, does possess a minimal lagrangian submanifold. To see this notice that $S U(2+2 k) / S(U(2) \times U(2 k)) \cong G_{2,2 k+2}(\mathbb{C})$, the complex grassmannian of complex 2-planes in $\mathbb{C}^{2 k+2}$. Hence the grassmannian $G_{2,2 k+2}(\mathbb{R})$ of real 2-planes in $\mathbb{R}^{2 k+2}$ is a lagrangian submanifold which as shown in 
$[22]$ is globally minimal. Notice that $G_{2,2 k+2}(\mathbb{C})$ also has Kähler submanifolds. Indeed, it is a compact homogeneous Kähler manifold and it is shown in [19] that any such manifold has a cellular decomposition in terms of analytic cycles. Hence for any $k$, the Kazama-Suzuki model with target $G_{2,2 k+2}(\mathbb{C})$ possesses both types of D-branes.

Let us conclude with a remark. We have obtained that the supersymmetric cycles of Kazama-Suzuki models are given by a distinguished set of submanifolds, which are "locally calibrated", yet not globally volume-minimising. We have also argued that this does not conflict with the BPS requirement coming from the DBI action. In order to make clearer the connection between the (geometric) analysis of supersymmetry and the one of dynamics, one would need a better understanding of the geometric content of the DBI action. One can easily analyse in the limit in which the $B$-field vanishes. This limit is to be taken in the Inönü-Wigner sense [23], as the level is sent to infinity. Looking at how the level enters in the expression for the energymomentum tensor, we see that this limit corresponds to the large volume limit in which, since the model is now given by free bosons on a circle, the geometry of the target space becomes toroidal. In this case, the supersymmetric cycles which we have found are indeed trivially calibrated. One can argue that the BPS condition remains true since this should not depend on the moduli, but we have no right to expect the same for the calibrated condition.

\section{Abelian T-Duality}

In this section we will set up the study of abelian $\mathrm{T}$-duality for the D-brane configurations we have analysed so far. For this purpose we will make use of the fact that Kazama-Suzuki models can be realised as gauged supersymmetric WZW models [24, 14].

Let us start by briefly reviewing T-duality symmetry for WZW models (for details see $[25,26,27]$ ). The structure of the duality group here is slightly different from the one present in flat backgrounds. First of all the WZW model is self-dual under an abelian T-duality transformation. Moreover T-duality symmetry is realised at the level of the affine algebra as Weyl transformations acting on the primary currents. Thus the symmetry underlying $\mathrm{T}$-duality is the invariance under the affine Weyl group.

T-duality for coset models (gauged WZW models) is intimately related to the T-duality symmetry of the original WZW models. In general one can consider two limiting cases, according to whether the gauged subgroup $H$ is semisimple or abelian. If $H$ is semisimple then the duality symmetry of the coset is inherited from that of the original WZW model: any dual pair of coset models is obtained by gauging a dual pair of WZW actions. In our case, since we want $G / H$ to be Kähler, we only consider subgroups such that rank $\mathfrak{g}=\operatorname{rank} \mathfrak{h}$. The 
duality transformations of the coset model are then obtained by considering those duality transformations at the level of the original theory which act trivially at the level of $H$. (In other words we consider only those Weyl transformations which act trivially on the $\mathfrak{h}$ currents.) This implies that a duality transformation on the Kazama-Suzuki model will act trivially on the Cartan subalgebra of $\mathfrak{h}$ which, assuming a regular embedding of $\mathfrak{h}$ in $\mathfrak{g}$, coincides with the one of $\mathfrak{g}$, thus leaving all the currents invariant. Hence we are only left with trivial T-duality transformations.

We will therefore consider coset theories where the gauged subgroup is abelian, hence of the form $G / U(1)^{\ell}$, where $\ell=$ rank $\mathfrak{g}$. In this case a dual pair of cosets consists in two theories obtained by performing either a vector or an axial gauging of a $U(1)$. The original affine Weyl symmetry of the WZW model guarantees that this axial-vector duality is an exact symmetry.

By a T-duality transformation we will understand a map, at the level of the fields, which preserves the $(2,2)$ superconformal structure of the model. More precisely, we will consider a map that acts trivially on the antiholomorphic sector of the theory and is given by an automorphism of $N=2$ SCA (whose automorphism group is $O(2)$ ) on the holomorphic sector. On the other hand, one can argue that if the original and the dual theories are to describe the same physics then they should couple in the same way to the gauge fields, and therefore the $\mathrm{T}$-duality transformation should fix the $N=1$ subalgebra generated by $\left(\mathrm{T}, \mathrm{G}_{0}\right)$. This leaves us with a $\mathbb{Z}_{2} \times \mathbb{Z}_{2}$ group of transformations, that basically distinguishes two types of $\mathrm{T}$-duality, which we will denote by $T_{A}$ and $T_{B}$, respectively:

$$
\begin{aligned}
\mathrm{T} & \rightarrow \mathrm{T}, & \mathrm{T} & \rightarrow \mathrm{T}, \\
\mathrm{G}_{0} & \rightarrow \pm \mathrm{G}_{0}, & \mathrm{G}_{0} & \rightarrow \pm \mathrm{G}_{0} \\
\mathrm{G}_{1} & \rightarrow \mp \mathrm{G}_{1}, & \mathrm{G}_{1} & \rightarrow \pm \mathrm{G}_{1}, \\
\mathrm{~J} & \rightarrow-\mathrm{J}, & \mathrm{J} & \rightarrow \mathrm{J} .
\end{aligned}
$$

$T_{A}$-duality is what one usually calls mirror symmetry $[18,17]$, but in our context we will treat both types of $\mathrm{T}$-dualities on an equal footing.

At the level of the current algebra this T-duality map acts as a Weyl transformation on the holomorphic currents. In our basis this will be described by a linear transformation

$$
I_{a}^{\prime}=T_{a}^{b} I_{b}, \quad \Psi_{a}^{\prime}=U_{a}^{b} \Psi_{b}
$$

while leaving invariant the currents in the antiholomorphic sector

$$
\bar{I}_{a}^{\prime}=\bar{I}_{a}, \quad \bar{\Psi}_{a}^{\prime}=\bar{\Psi}_{a} .
$$

In this way the antiholomorphic SCA will be trivially preserved; for the holomorphic sector we impose that the T-duality map at the level of 
the currents will induce a $T_{A}$ or $T_{B}$ transformation of the corresponding SCA.

The analysis of the linear maps $T$ and $U$ is completely analogous to the one of the matrices $R$ and $S$ describing the boundary conditions. Since the essential symmetry here is the Weyl symmetry of the ungauged WZW model, we start by defining the T-duality transformation on the $\mathfrak{g}$ currents and impose that it preserve the $N=1$ algebra (1)-(3). From this we obtain that $U= \pm T$, that $T$ preserves the metric $\Omega$ and gives rise to a Lie algebra automorphism on $\mathfrak{g}$. From the transformation law of $\mathrm{G}_{0}$ we also deduce that $T$ has a block diagonal structure with respect to $\mathfrak{h}$.

Since T-duality is realised as Weyl transformations on the currents it follows that the orthogonal matrix $T$ can be taken to be symmetric and such that $T^{2}=\mathbb{1}$. In the previous sections we have seen that the requirement for a boundary state to have a geometric interpretation as a D-brane implies that the matrix $R$ defining that boundary state is symmetric and squares to the identity matrix. If we now require that also the T-dual configuration defined by the matrices $\tilde{R}$ and $\tilde{S}$

$$
I_{a}^{\prime}(z)=\tilde{R}_{a}^{b} \bar{I}_{b}^{\prime}(\bar{z}), \quad \Psi_{a}^{\prime}(z)=\tilde{S}_{a}^{b} \bar{\Psi}_{b}^{\prime}(\bar{z}),
$$

have such a geometric interpretation, then we can deduce that the T-duality transformation commutes with the original D-brane configuration, $R T=T R$. In other words $R$ and $T$ are simultaneously diagonalisable.

We now analyse the two types of duality transformations separately. Notice first that $T_{A}$ maps from one type of boundary condition to the other. Furthermore, from the action of $T_{A}$ on $\bar{G}_{1}$ we obtain

$$
A^{\alpha \beta} T_{\alpha}^{\gamma} T_{\beta}^{\delta}=-A^{\gamma \delta}
$$

or, in other words, $T$ and $A$ anticommute. From this one can deduce that the order of the $T$ transformation, by which we mean the number of $(-1)$ eigenvalues, is

$$
\operatorname{ord} T_{A}=\frac{1}{2} \operatorname{dim} G / H .
$$

We therefore consider two different cases according to the dimension of the coset space:

(i) $\operatorname{dim} G / H=4 k$;

In this case ord $T_{A}=2 k$, and hence such a $\mathrm{T}$-duality transformation will map within the same Type II theory. For example, if we start with a configuration corresponding to a D-brane which wraps around a $2 k$-dimensional A-type cycle $\gamma$, and we perform a $T_{A}$-duality transformation, we end up with a configuration where a D-brane wraps around an even dimensional B-type cycle $\gamma^{\prime}$. On the other hand, the $T_{A}$-dual of an even dimensional cycle (corresponding to a holomorphic submanifold) will be a $2 k$-dimensional 
cycle corresponding (in the HSS case) to a lagrangian submanifold. This is summarised in Table 1.

\begin{tabular}{|c|c|c|c|}
\hline IIA & IIB & $\begin{array}{c}\text { A-type } \\
\operatorname{dim} \gamma=2 k\end{array}$ & $\begin{array}{c}\text { B-type } \\
\operatorname{dim} \gamma=0,2, \ldots, 4 k\end{array}$ \\
\hline IIA & IIB & B-type & A-type \\
& & $\operatorname{dim} \gamma^{\prime}=0,2, \ldots, 4 k$ & $\operatorname{dim} \gamma^{\prime}=2 k$ \\
\hline
\end{tabular}

TABLE 1. Effect of a $T_{A}$ duality transformation of order $2 k$ : the top row gets mapped to the bottom one.

(ii) $\operatorname{dim} G / H=4 k+2$;

In this case ord $T_{A}=2 k+1$, and therefore $T_{A}$ will map from Type IIA to Type IIB and vice versa. The $T_{A}$-dual of a $(2 k+$ 1)-dimensional A-type cycle will be an even dimensional B-type cycle, and vice versa. This is summarised in Table 2 .

\begin{tabular}{|c|c|c|c|}
\hline IIA & IIB & $\begin{array}{c}\text { A-type } \\
\operatorname{dim} \gamma=2 k+1\end{array}$ & $\begin{array}{c}\text { B-type } \\
\operatorname{dim} \gamma=0,2, \ldots, 4 k+2\end{array}$ \\
\hline IIB & IIA & B-type & A-type \\
& & $\operatorname{dim} \gamma^{\prime}=0,2, \ldots, 4 k+2$ & $\operatorname{dim} \gamma^{\prime}=2 k+1$ \\
\hline
\end{tabular}

TABLE 2. Effect of a $T_{A}$ duality transformation of order $2 k+1$ : the top row gets mapped to the bottom one.

Finally, we turn now to the $T_{B}$-duality map. This maps between boundary states of the same type. Moreover, if we consider its action on $\mathrm{G}_{1}$ which implies

$$
A^{\alpha \beta} T_{\alpha}^{\gamma} T_{\beta}^{\delta}=A^{\gamma \delta},
$$

we obtain for the order of $T_{B}$

$$
\operatorname{ord} T_{B}=0,2,4, \ldots, \operatorname{dim} G / H,
$$

from where we can conclude that it also maps within the same Type II theory. If we start with a configuration corresponding to a D-brane wrapping around an even dimensional B-type cycle $\gamma$, and we perform a $T_{B}$-duality transformation, we end up with a configuration where a D-brane wraps around another even dimensional B-type cycle $\gamma^{\prime}$. The other possibility is to start with an A-type cycle $\gamma$ of dimension $\frac{1}{2} \operatorname{dim} G / H$ : in this case the $T_{B}$-dual cycle $\gamma^{\prime}$ will be will be of the same dimension, which means that $T_{B}$ has to act nontrivially in an equal number of Neumann and Dirichlet dimensions. The effect of a $T_{B}$ duality transformation is summarised in Table 3.

One has to stress that all these T-dual pairs are "subject to availability". By this we mean that the existence of the corresponding cycles $\gamma$ 


\begin{tabular}{|c|c|c|c|}
\hline IIA & IIB & A-type & B-type \\
& & $\operatorname{dim} \gamma=\frac{1}{2} \operatorname{dim} G / H$ & $\operatorname{dim} \gamma=0,2, \ldots, \operatorname{dim} G / H$ \\
\hline IIA & IIB & A-type & B-type \\
& & $\operatorname{dim} \gamma^{\prime}=\frac{1}{2} \operatorname{dim} G / H$ & $\operatorname{dim} \gamma^{\prime}=0,2, \ldots, \operatorname{dim} G / H$ \\
\hline
\end{tabular}

TABLE 3. Effect of a $T_{B}$ duality transformation: the top row gets mapped to the bottom one.

in the coset manifold is not guaranteed a priori and has to be looked at case by case.

\section{ACKNOWLEDGEMENTS}

It is a pleasure to thank Ralph Blumenhagen, Michael Flohr, Beatriz Gato-Rivera, Christoph Schweigert and Arkady Tseytlin for valuable discussions. In addition I would like to thank José Figueroa-O'Farrill for getting me interested in this topic and for a critical reading of the paper. I would also like to thank the referee for useful criticism of an earlier version of this paper. Finally, I would like to acknowledge the support of the Isaac Newton Institute for Mathematical Sciences, Cambridge, where this work was started.

\section{REFERENCES}

[1] K. Becker, M. Becker, and A. Strominger, "Fivebranes, membranes and non-perturbative string theory," Nuc. Phys. 456 (1995) 130. hep-th/9509175.

[2] H. Ooguri, Y. Oz, and Z. Yin, "D-branes on Calabi-Yau spaces and their mirrors," Nuc. Phys. B477 (1996) 407-430. hep-th/9606112.

[3] K. Becker, M. Becker, D. R. Morrison, H. Ooguri, Y. Oz, and Z. Yin, "Supersymmetric cycles in exceptional holonomy manifolds and Calabi-Yau 4-folds," Nuc. Phys. B480 (1996) 225. hep-th/9608116.

[4] F. R. Harvey and H. B. Lawson, "Calibrated geometries," Acta Math. 148 (1982) 47-157.

[5] F. R. Harvey, Spinors and calibrations. Academic Press, 1990.

[6] M. Bershadsky, V. Sadov, and C. Vafa, "D-branes and topological field theory," Nuc. Phys. B463 (1996) 420. hep-th/9511222.

[7] M. Kato and T. Okada, "D-branes on group manifolds," Nuc. Phys. B499 (1997) 583. hep-th/9612148.

[8] M. Blau and G. Thompson, "Aspects of $N_{T} \geq 2$ topological gauge theories and D-branes," Nuc. Phys. B492 (1997) 545-590. hep-th/9612143.

[9] M. Blau and G. Thompson, "Euclidean SYM theories by time reduction and special holonomy manifolds," Phys. Lett. B415 (1997) 242. hep-th/9706225.

[10] B. S. Acharya, J. M. Figueroa-O'Farrill, M. O'Loughlin, and B. Spence, "Euclidean D-branes and higher-dimensional gauge theory," Nuc. Phys. B514 (1998) 583-602. hep-th/9707118.

[11] Y. Kazama and H. Suzuki, "Characterization of $N=2$ superconformal models generated by coset space method," Phys. Lett. B216 (1989) 112.

[12] Y. Kazama and H. Suzuki, "New $N=2$ superconformal field theories and superstring compactification," Nuc. Phys. B321 (1989) 232. 
[13] C. Schweigert, "On the classification of $N=2$ superconformal coset theories," Comm. Math. Phys. 149 (1992) 425.

[14] J. M. Figueroa-O'Farrill and S. Stanciu, "Nonreductive WZW models and their CFTs. II: $N=1$ and $N=2$ cosets," Nuc. Phys. B484 (1997) 583-608. hep-th/9605111.

[15] W. Lerche, C. Vafa, and N. P. Warner, "Chiral rings in $N=2$ superconformal theories," Nuc. Phys. B324 (1989) 427.

[16] N. Ishibashi, "The boundary and crosscap states in conformal field theories," Mod. Phys. Lett. A4 (1989) 251.

[17] A. Strominger, S. T. Yau, and E. Zaslow, "Mirror symmetry is T-duality," Nuc. Phys. B479 (1996) 243-259. hep-th/9606040.

[18] D. R. Morrison, "Mirror symmetry and the type II string," Nucl. Phys. Proc. Suppl. 46 (1996) 146-155. hep-th/9512016.

[19] A. Borel, "Kählerian coset spaces of semi-simple Lie groups," Proc. Nat. Acad. Sci. USA 40 (1954) 1147-1151.

[20] H. Ishikawa, Y. Matsuo, Y. Sugawara, and K. Sugiyama, "BPS mass spectrum from D-brane action." hep-th/9605023.

[21] Y. Sugawara and K. Sugiyama, "D-brane analyses for BPS mass spectra and U-duality." hep-th/9707205.

[22] L. H. Van, "Minimal surfaces in homogeneous spaces," Math. USSR Izvestiya 32 (1989) 413-427.

[23] E. Inönü and E. P. Wigner, "On the contraction of groups and their representations," Proc. Nat. Acad. Sci. USA 39 (1956) 510-524.

[24] E. Witten, "The $N$-matrix model and gauged WZW models," Nuc. Phys. B371 (1992) 191. hep-th/9606040.

[25] E. Kiritsis, "Exact duality symmetries in CFT and string theory," Nuc. Phys. B405 (1993) 109-142. hep-th/9302033.

[26] E. Alvarez, L. Alvarez-Gaumé, and Y. Lozano, "A canonical approach to duality transformations," Phys. Lett. B336 (1994) 183-189. hep-th/9406206.

[27] A. Giveon and E. Witten, "Mirror symmetry as a gauge symmetry," Phys. Lett. B332 (1994) 44-50. hep-th/9404184.

Theoretical Physics Group

BLACKETT LABORATORY IMPERIAL COLLEGE Prince CONSORT ROAD LONDON SW7 2BZ, UK 